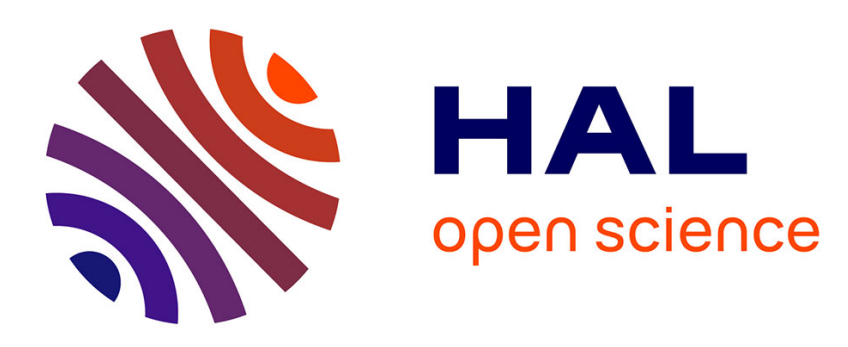

\title{
Wireless Connectivity of CPS for Smart Manufacturing: A Survey
}

Ahmadzai Ahmadi, Maisam Moradi, Chantal Cherifi, Vincent Cheutet, Yacine Ouzrout

\section{- To cite this version:}

Ahmadzai Ahmadi, Maisam Moradi, Chantal Cherifi, Vincent Cheutet, Yacine Ouzrout. Wireless Connectivity of CPS for Smart Manufacturing: A Survey. 12th IEEE International Conference on Software, Knowledge, Information Management and Applications (SKIMA 2018), Dec 2018, Phnom Penh, Cambodia. 8 p. hal-01952355

\section{HAL Id: hal-01952355 https://hal.science/hal-01952355}

Submitted on 12 Dec 2018

HAL is a multi-disciplinary open access archive for the deposit and dissemination of scientific research documents, whether they are published or not. The documents may come from teaching and research institutions in France or abroad, or from public or private research centers.
L'archive ouverte pluridisciplinaire HAL, est destinée au dépôt et à la diffusion de documents scientifiques de niveau recherche, publiés ou non, émanant des établissements d'enseignement et de recherche français ou étrangers, des laboratoires publics ou privés. 


\title{
Wireless Connectivity of CPS for Smart Manufacturing:
}

\author{
A Survey \\ Ahmadzai AHMADI, Maisam MORADI, Chantal CHERIFI, Vincent CHEUTET, Yacine OUZROUT \\ University Lyon, University Lumiere Lyon2, INSA Lyon, DISP EA4570, France \\ \{Ahmadzai.Ahmadi, Chantal.BonnerCherifi, yacine.ouzrout\}@univ-lyon2.fr, vincent.cheutet@insa-lyon.fr
}

\begin{abstract}
Fourth Industrial revolution (Industry 4.0) has entirely reshaped the landscape of the global world from both enterprise and academia aspect. In the meantime Cyber-Physical System (CPS) has proven its existence by effectively monitoring, analyzing, automating and communicating with every corner. So, wireless connectivity in the industrial world is considered as the challenging issues to be tackled. Sensor based technologies in association with wireless communication are playing main role in Industry 4.0 and CPS environment to make the whole world as smart, sharp and dynamic place. Thus, smart factories must be built by introducing the sensor enabled communication entities such as, CPS, Wireless Sensor Networks (WSNs) with strong and reliable network connection. So, this paper selects the suitable wireless connectivity in Industry 4.0 by focusing at network metrics for instance, latency, reliability, longevity, and throughput. Besides, the critical wireless needs of the CPS based Industry 4.0 are observed and verified through rigorous literature than wireless network and communication technology is proposed from the pool of classical methods. That proposed wireless networks and technology will lead to the future CPS problems in the light of any standard.
\end{abstract}

Keywords- CPS, Smart, manufacturing, Wireless Communication, Wireless Sensor Network.

\section{INTRODUCTION}

The existence of wireless technology has brought ease to various human activities, and solved problems regarding communication, business, productivity, industry, mobility. Indeed, electromagnetic waves are the media used to transmit information over of the wireless network. The main functionality of the wireless networks is performed by the radio communication. There are two broad classes of wireless technologies short-range and long-range respectively. Former is defined by Wireless Sensor Networks (WSNs), which consists of spatially distributed autonomous devices using sensors to monitor physical or environmental conditions. Also, Industrial Wireless Networks (IWNs) lies in the umbrella of WSN. While later explains the fiber, optic based local area networks, long haul networks, etc. IWSNs are defined as a collection of distributed wireless devices, and wirelessly communicate to measure, monitor and control physical environments of the industry [1]. Wireless networks play a key role to make possible the deployment of the fourth industrial revolution, namely Industry 4.0. Industry 4.0 aims to inter-connect and computerize the traditional industry and make it smart by being underpinning by a set of technologies such as Cyber-Physical Systems (CPSs), the Internet of Things (IoT), cloud computing, big data analytics, information and communication technologies and cognitive computing [2]. CPS is considered as the emerging component for Industry 4.0. Detailed explanation of the wireless technology-based CPS architecture with strong connectivity between sensors and related parts is shown in Fig.1[3].

One of the critical challenge faced by CPS in this emerging trend of industrial period is to achieve high yield and products by dynamic structure and intelligent sensor based technologies. Enhancing the product quality is one the emerging ingredient in every sector, so it is very vital to pay more focus to handle this challenge in the CPS and the Industry 4.0. Flexible and reconfigurable production systems and effective human-machine interaction to reduce the time and cost of machine control and maintenance is always inevitable. This, in turn, bring forth the issue of employee adaptation to the new technology. In Industry 4.0, real-time data acquisition, storage, and data analysis using machine learning techniques become formidable tasks. Moreover, uncertainties in the quality and volume of product returns [3] become an unresolvable issue. It requires an effective data and storage management for intelligent monitoring and intelligent control and increases collaboration in productivity [4]. In this connection, many researchers are moving towards cloud management. In turn, this imposes three key problems for example, virtualizing resource management, scheduling of cloud resources, and Life Cycle Management (LCM). Improve production also depends on the seamless data exchange pattern from physical component to cyber component. Physical-to-cyber interface processes history, traceability, and tracking to rectify processing defects and product recalls. It needs more than just using off-the-shelf RFID systems. Furthermore, conventional methods become formidable difficulties to specify and verify machine-to machine interaction behaviors among industrial equipment [6].

Information Technology (IT) is an important part of CPS for manufacturing. This is characterized from the two critical issues i.e., human-centered and cyber-centered platforms in the industry 4.0. The solutions used so far to control and optimize production are incapable of solving existing problems. It requires Holistic Production Control (HPC). Human-centric IT development requires to succeeding the emerging CPS for manufacturing in Industry4.0 [7]. Due to the personalized product and new modular, flexible, adaptive, and reconfigurable manufacturing paradigm, the smart space infrastructure changes quickly. Therefore, the factory often requires broader skills of a human worker to 

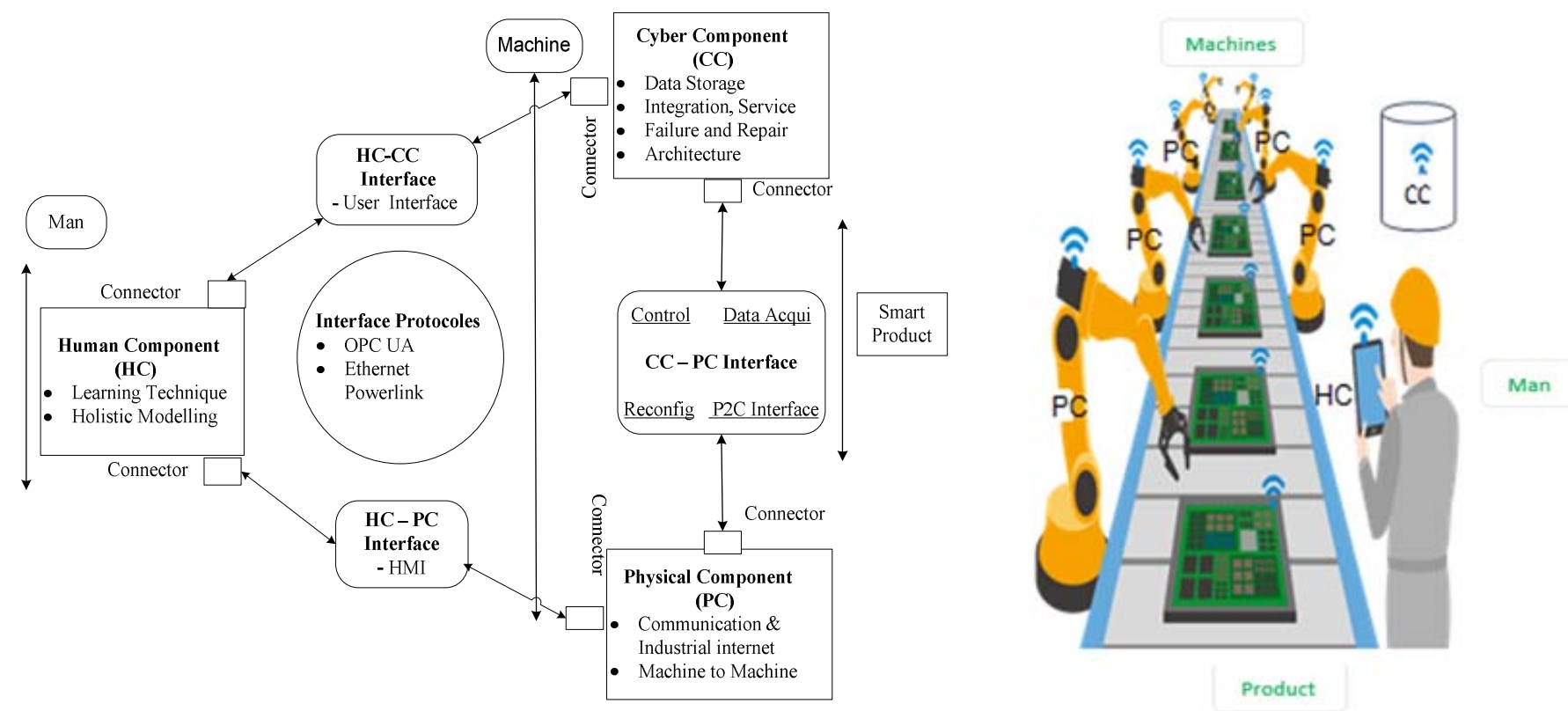

Fig.1. CPS Architecture for Smart Manufacturing

understand and manage diverse interrelation between a physical object and digital counterparts.

For dynamic reconfiguration, adaptive and flexible systems are the need of the hour due to the rapid progress in the technology. It requires a more advanced approach to merging different processes and specific dynamic products with quality monitoring, fault detection, to achieve maximum productivity [8], [9]. The new product ramp up often causes different unpredicted failures that demand formidable failure management system to guarantee the planned time-to-market. Whenever a failure is detected, repair becomes an in- evitable task for ensuring long lifecycles. New automation and a high degree of flexibility in a repair of high-value parts require a substantial amount of manual preparation for repair process chain [10], which is a bottleneck to stop it from final success, besides a value chain risk assessment is an important issue.

Uniform standard is the common language for the all products and systems to exchange information and information. So, it is the dire need to develop the state-of-theart CPS enabled industrial environment with dynamic connectivity and strong capabilities to monitor and examine the system. for manufacturing is an emerging technology in the industry. A few researches address the challenges of standardization and seamless process integration, seamless data aggregation and disaggregation, standardization compliance, and product-service innovation, product variety, quality standards, support services, and immediacy or order satisfaction. Industrial automation systems (IASs) are commonly developed using the defector standard IEC 61131. Although version 2.0 of IEC 61131 is introduced to address the new challenges of Complex Industrial Automation (CIA) systems, the standard IEC 61499 has been defined to eliminate limitation of IEC 61131. However, we need more and more work on standardization for maturing this new emerging technology [11].

That connectivity shows a vital role in the transfer of collected data in sensor network-based industrial IoT systems[12]. The sensors and actuators are the intelligent and dynamic entities to monitor and manage the entire industrial platform, while wireless communication is not possible without smart and sensor-based components for the strongly connected industrial and enterprise world. Because it is easy to share the knowledge and information between interconnected entities. Above all wireless connectivity among interconnected smart world in the industrial landscape is one of the key challenges to be considered in this research. Besides, wireless technologies and reliable strong connectivity are the cornerstones for the CPS and Industry 4.0 in association with distinct network metrics i.e., latency, reliability, longevity, and throughput.

The main contribution of this paper is to identify the wireless standards for CPS wireless connectivity. We followed the wireless requirements of CPS for Industry 4.0 and make a parallel with the features of existing standards. To go a step beyond, we draw some challenges and solutions for CPS wireless connectivity. The remaining of the paper is organized as follows. Section 2, related work Section 3, Wireless Connectivity Technologies \& Standards for CPS. Section 4, Future Challenges and Solution for CPS Wireless Connectivity Paper is concluded in the Section 5.

\section{RELATED WORKS}

In this section, we will focus on three topics Industry 4.0, CPS and wireless connectivity. Technology has played a major role in advancing most areas of our everyday lives and it can also have a significant effect in the world of industry. 
Developments in information technology, mobile communications and robotics have led to the growing use of digital technologies in factories around the world. Industrial companies can now create extensive data communication networks in the harshest of environments while achieving low costs, low latency, longevity and reliability.

\section{A. Industry 4.0}

Considering industry is a core element of the value chain and a crucial component in the technological development, job creation and economic stability of a country, the traditional industrialized countries have assumed a leading role in the fourth industrial revolution [13]. We analyzed the latest documents on the topic of "smart manufacturing and Industry 4.0 " according to year of published, country and subject area, as represented in Table 1.

In the context of Industry 4.0, manufacturing systems are updated to an intelligent level. Intelligent manufacturing takes advantage of advanced information and manufacturing technologies to achieve flexible, smart, and reconfigurable manufacturing processes in order to address a dynamic and global market [14]. It enables all physical processes and information flows to be available when and where they are needed across holistic manufacturing supply chains, multiple industries, small and medium-sized enterprises (SMEs), and large companies [15]. Intelligent manufacturing requires certain underpinning technologies in order to enable devices or machines to vary their behaviors in response to different situations and requirements based on past experiences and learning capacities [16]. These technologies enable direct communication with manufacturing systems. The concept of Industry 4.0 was initially proposed for developing German economy in 2011 [17][18]. The first industrial revolution launched at the end of the 18th century. It was represented by mechanical production plants based on water and steam power; the second industrial revolution started at the beginning of the 20th century with the symbol of mass labor production based on electrical energy; the third industrial revolution started in the 1970s with the characteristic of automatic production based on electronics and internet technology; the fourth industrial revolution, namely Industry 4.0 , is ongoing, with the characteristics of CPS production, based on heterogeneous data and knowledge integration. The main roles of CPS are to fulfill the agile and dynamic requirements of production, and to improve the effectiveness and efficiency of the entire industry. Industry 4.0 encompasses numerous technologies and associated paradigms, including Radio Frequency Identification (RFID), Enterprise Resource Planning (ERP), Internet of Things (IoT), cloud-based manufacturing, and social product development [19][20].

From the analysis of manufacturing methods, it appears that there is a dire need to answer this key questions. How SMEs are build and setting-up from conventional manufacturing practices to the smart manufacturing/Industry 4.0? The traditional manufacturing and smart manufacturing
Table.1 Published documents by year, county and subject area about "Smart Manufacturing and Industry 4.0". Source: [www.scopus.com] [30 June 2018].

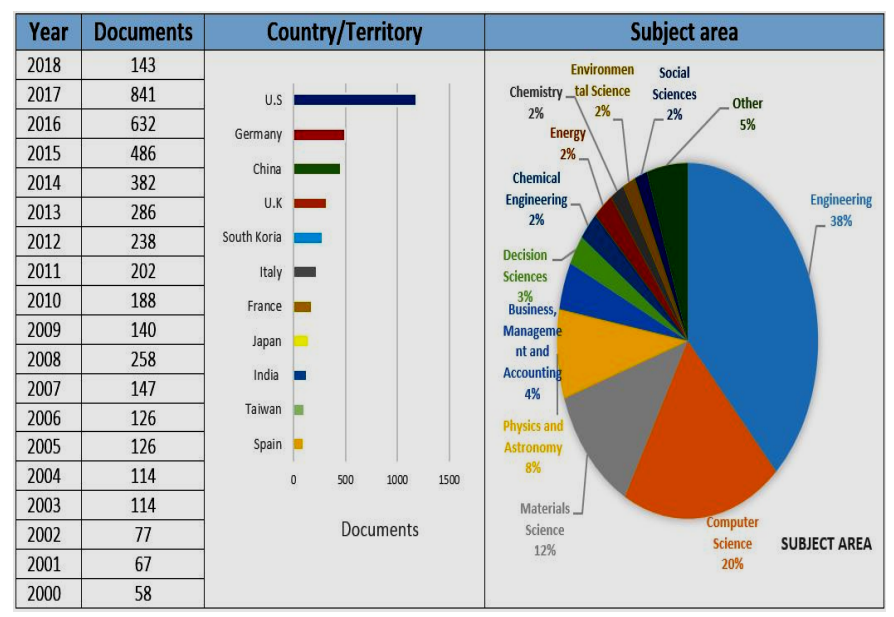

are compared based on technologies and tools as shown in the Table 2. In addition, various entities, data sources, performance metrics and technologies are presented with the extensive detail to portray the clear image and importance of the smart manufacturing.

\section{B. Cyber-physical systems}

As mentioned previously, CPS are one of the key technologies for Industry 4.0. CPSs are smart systems that depend on the collaboration of cyber and physical components. They link the physical world through sensors, actuators, robotics, and embedded systems with the virtual world of information processing. The term CPS first appeared in 2006 when an Network Files System (NFS) workshop was held in Austin, Texas, USA [3]. It was defined as "a system composed of collaborative entities, equipped with calculation capabilities and actors of an intensive connection with the surrounding physical world and phenomena, using and providing all together services of treatment and communication of data available on the network.

Table.3 Published documents by Year, county and subject area about CPS. Source: [www.scopus.com] [30 June 2018].

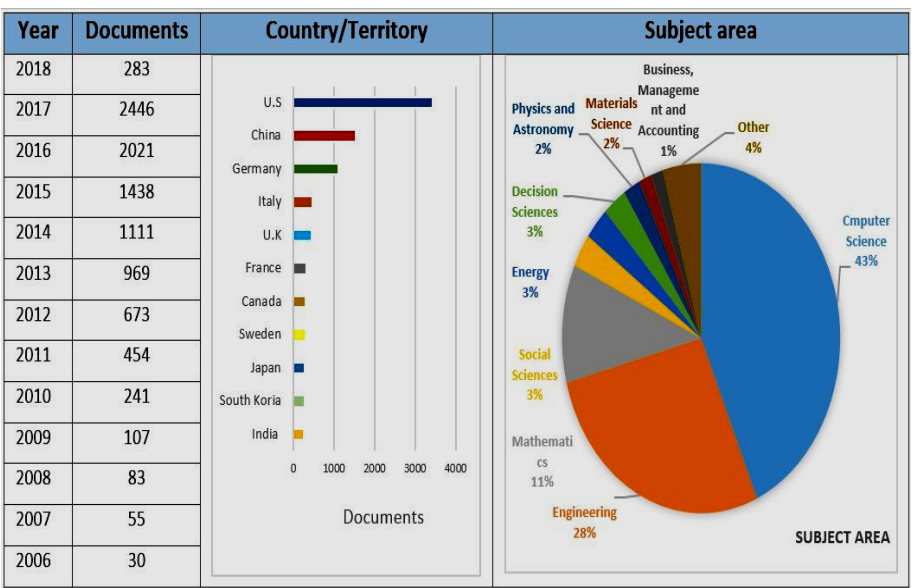


Table.2 Comparison between traditional and smart manufacturing.

\begin{tabular}{|c|c|c|c|c|c|}
\hline Entity & Data Source & Traditional Manufacturing & \multicolumn{2}{|c|}{ Smart Manufacturing } \\
\cline { 2 - 5 } & Metrics & Technologies & Metrics & Technologies \\
\hline Parameters & $\begin{array}{c}\text { Connectors, Sensors and } \\
\text { Actuators }\end{array}$ & Accuracy & Manual operation & $\begin{array}{c}\text { Self-driven and } \\
\text { predictive }\end{array}$ & $\begin{array}{c}\text { Delay-tolerant and } \\
\text { high outcome based }\end{array}$ \\
\hline Machine & Adaptive Controller & Production & $\begin{array}{c}\text { Condition based } \\
\text { maintenance }\end{array}$ & $\begin{array}{c}\text { Flexible and } \\
\text { scalable }\end{array}$ & $\begin{array}{c}\text { Adaptive and } \\
\text { Autonomous }\end{array}$ \\
\hline $\begin{array}{c}\text { Production } \\
\text { System }\end{array}$ & Heterogeneous networks & Complexity & $\begin{array}{c}\text { High resource } \\
\text { consumption }\end{array}$ & $\begin{array}{c}\text { Economical and } \\
\text { effective }\end{array}$ & High connectivity \\
\hline
\end{tabular}

In order to integrate CPS inside a production system, a clear methodology should be defined in order to support manufacturers to establish their evolution strategy. Such a methodology is up to now not available in the literature. A first step mandatory for this methodology is to choose a representation of CPS suited to their integration in-side a production system. In the literature, different CPS architectures for smart manufacturing have been proposed [3]. The goal of those architectures are to build the CPS for smart factories. Up to now, one can distinguish the (5C, 8C and $3 \mathrm{C}$ architectures). It is reviewed the CPS documents by date of publication, country and subject area, as represented in Table 3. In literature, the main challenges that have been highlighted (improvement in production, dynamic reconfiguration, information technology, standardization and CPS wireless connectivity). In our previous work [11] we focused on the standardization aspect. Here, we intend to target the question of wireless connectivity.

\section{Wireless Connectivity of smart manufacturing and CPS technology}

In the context of Industry 4.0, data (or information) and wireless communications is transmitted through IWNs. Big data, industrial clouds, and industrial systems are used in Industry 4.0 to increase productivity, reduce cost and energy consumption, and enhance flexibility and personalization. To realize the objectives of Industry 4.0, there are new requirements for the whole IWNs system, especially for the service and the data. The data carries important information for the factory, services, manufacturing, and users and wireless communication is responsible for transmitting and receiving this data. In the communication part of IWSs, QoS is one of the important thing that we should focus on QoS because the QoS of IWNs include real-time, reliability, longevity, security and privacy criteria [1].

Table.4 Requirements of CPS based on wireless for M2M communication in smart manufacturing.

\begin{tabular}{|l|c|c|c|}
\hline Requirements & $\begin{array}{c}\text { Motion } \\
\text { Control }\end{array}$ & $\begin{array}{c}\text { Condition } \\
\text { Monitoring }\end{array}$ & $\begin{array}{l}\text { Augmented } \\
\text { Reality }\end{array}$ \\
\hline Latency/Cycle Time & $\begin{array}{c}250 \mu \mathrm{s}- \\
1 \mathrm{~ms}\end{array}$ & $<100 \mathrm{~ms}$ & $<10 \mathrm{~ms}$ \\
\hline Reliability (PER) & $1 \mathrm{e}-8$ & $1 \mathrm{e}-5$ & $1 \mathrm{e}-5$ \\
\cline { 2 - 4 } & \multicolumn{3}{|c|}{$99-99.99 \%$} \\
\hline $\begin{array}{l}\text { Longevity (Battery } \\
\text { Life time) }\end{array}$ & $\mathrm{n} / \mathrm{a}$ & 10 years & 1 day \\
\hline Data rate & Gbit/s & Kbit/s & Gbit/s \\
\hline
\end{tabular}

Wireless communication represents a key enabling technology for the smart industry systems, which will be characterized by an unprecedented degree of flexibility, versatility, usability and efficiency. However, many industrial applications have highly demanding requirements with respect to reliability, latency and real-time capability. For that reason, existing wireless technologies are often not able to adequately support corresponding use cases and applications. For selecting a wireless network for CPS in industry 4.0, first we need to understand what are the requirements of CPS bases on wireless? After discovering the requirements, we considered

to the existent wireless networks to find which of existent wireless networks can address in a good way to these requirements Table 4 [21][22].

Machine-to-machine (M2M) communication plays a critical component of Industry 4.0 and thus resulting in Internet or IoT. Industry 4.0 will involve the coordination between disparate devices that are physically placed in distant geographical locations and across different industries. Both IoT and CPS form the technology basis for Industry 4.0. The communication requirement in Industry 4.0 is focused on multiple factors such as reliability, latency, longevity of communicating devices as opposed to only the throughput, a traditional focus of human centric wireless communication systems. Most devices within a factory are currently connected based on wired infrastructure working over industrial protocols. However, the wireless solutions are increasingly playing a role to wired solutions. The factors accelerating the adoption of wireless in industrial scenario include the ease of deployment and scalability especially for a very large area of coverage. Wireless solutions are suitable also in hard to reach locations/remote locations with wires. The dynamic topology networks in addition to the mobility and efficient coordination between disparate geographic locations will play a key role in the adoption of wireless solutions in the Industry 4.0 are [21].

The IWNs technology is defined as a collection of distributed wireless devices which are communicating wirelessly in an industry to measure, monitor and control physical environments of industries. The Important technology which make possible to deployment industry 4.0 is IWNs Fig.2. IWNs have been gradually entering industry vision, and are becoming vital foundations for realizing the architecture of industry 4.0 and smart factories. In industry 4.0 IWNs are 


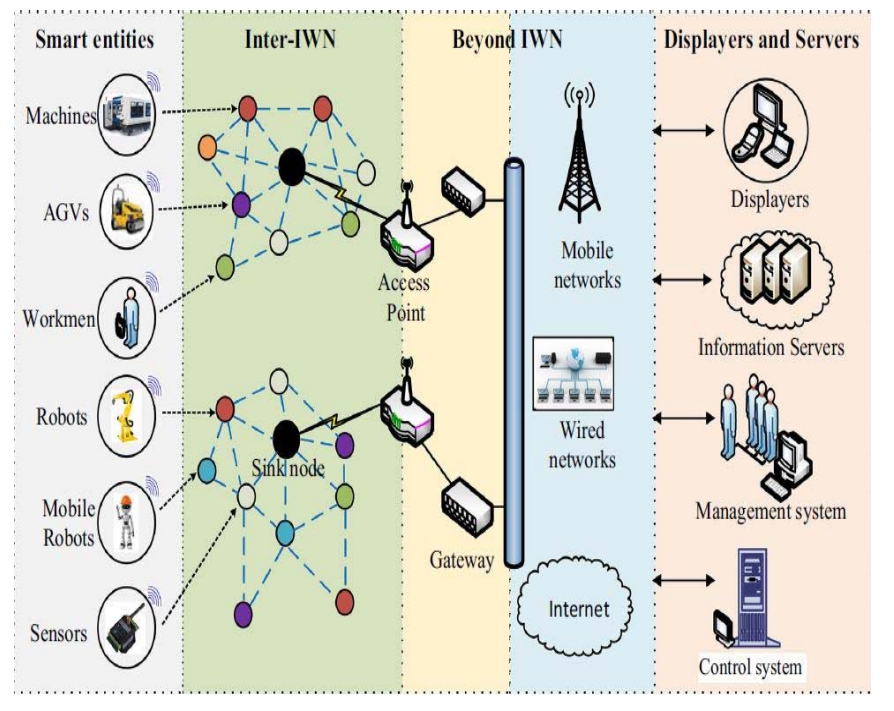

Fig.2. Industry wireless network schematic diagram [1]

different from traditional WSNs, the main differences between them can be latency, mobility, environments and capacity. As shown in fig.2, The IWN communications system divided into four components: smart entities, inter-IWNs, beyond IWNs, displayers and servers. Within IWNs, smart entities (such as workmen, AGVs, machines, ordinary sensors) with wireless transceivers could be regarded as wireless nodes. It is clear that IWNs are formed between nodes by wireless radios. Beyond IWNs, the access point nodes and the gateway create a bridge to other networks such as cellular, wired, internet and other public networks. Finally, higher level data applications including data servers, management, controllers, and displayers, may be based on these specific networks. In IWNs QoS indexes include real-time, reliability, longevity, security and privacy controls. Furthermore, QoS and QoD are influenced by each other. It can be concluded that IWNs play a primary role in the framework of Industry 4.0 and the smart factory. The Requirements of IWNs are Low latency required in IWNs application, High energy consumption and high cost, to achieve teal-time performance, Mobility: required more moving nodes such as moveable robots, automatic guided vehicles, unmanned aerial vehicles, workmen and so on, Operation Environment: to ensure reliability and efficient communication required more strategies, because IWNs operate in a challenging environments such as signal interferences, high temperature, vibration and etc, and High Capacity: IWNs modes required higher capacity to handle data processing, it means that IWS should be smarter[1].

\section{WIRELESS CONNECTIVITY TECHNOLOGIES \& STANDARDS FOR CPS}

In CPS, computing, communication and control technologies are tightly integrated and nearly all CPS systems are composed on the support of existing communication networks and standards. Therefore, it is important to find the right available wireless standards based on requirements of system. Therefore, flexibility in CPS allow to the designer to freely select the best combination of technologies for their systems. It is not possible to cover in single standard types of communications schemes and need of CPSs as well as types of matching communication technology options. Thus, different standards which integrate various technologies are required to cover different areas of the communication scope [23]. Our objective is to identify the wireless connectivity standards for industry 4.0 which satisfy the wireless requirements of CPS. Hereafter, the standards are discussed one by one.

\section{A. Zigbee}

ZigBee technology is a wireless networking protocol with low data rate, low power consumption and low cost, which was created for automation control and sensor networks based on IEEE 802.15.4 standard. ZigBee specification provided by ZigBee Alliance. ZigBee network can contains a huge number of nodes up to 65000, and connect them in any industry into single control network [23][24].

\section{B. Bluetooth}

Bluetooth is a short range wireless communication system with low power consumption which providing connectivity for many electronic devices. It was grown by Ericson, but run under the auspices of the Bluetooth Special Interest Group (SIG) which develops the Bluetooth standards, it has developed to provide faster speeds, greater flexibility and far more capability. Bluetooth confirmed as (IEEE 802.15.1) standard. Bluetooth technology has progressed significantly and has been expanded to provide not only the traditional short range audio streaming, to applications like mesh connectivity for CPSs, IoT and M2M communications. Bluetooth Low Energy standard updated to close the energyefficiency break among ZigBee and Bluetooth for no streaming sensor-node-type applications [23].

\section{C. $W i-F i$}

Wi-Fi (Wireless Fidelity) is a genetic term that refers to the IEEE 802.11 wireless communication standard for WLANs and works on physical and data link layer. The different variants like $802.11 \mathrm{a} / \mathrm{b} / \mathrm{g} / \mathrm{n}$ or $802.11 \mathrm{ad}$ are different standards within the overall series and they define different variants. By releasing updated variants, the overall technology has been able to keep pace with the ever growing requirements for more data and higher speeds, etc. There are two types of WLAN network that can be formed using a Wi-Fi system, infrastructure networks; and ad-hoc networks. The infrastructure application is aimed at office areas or to provide a "hotspot". A backbone wired network is still required and is connected to a server. The wireless network is then split up into a number of cells, each serviced by a base station or Access Point (AP) which acts as a controller for the cell. Each Access Point may have different ranges. The other type of networks that may be used is termed an Ad-Hoc network. As Wi-Fi has developed, many new variants or standards have been developed to accommodate the increasing speeds and performance. Along with the current low power technologies like LoWPAN, Bluetooth ZigBee, passive optical network and 802.11 WiFi standards are similarly making its way into the market with its own advantages in high range and improved 
speed. Beside with IEEE, WiFi Alliance has a new standard for the proximity applications. [23][24].

\section{LoRaWAN}

LoRa is a Long Range low power wireless standard aimed at providing a cellular style low data rate communications network and developed for IoT and M2M applications [28].

\section{E. Passive Optical Network (PON)}

Passive Optical Network (PON) is a system that brings optical fiber cabling and signals most of the way to the end user. Depending on where the PON terminates. A PON consists of an Optical Line Termination (OLT) at the communication Company's office and a number of Optical Network Units simply describes the fact that optical transmission has no power requirements or active electronic parts once the signal is going through the network [28].

\section{F. Mobile Cellular Networks}

Mobile cellular telecommunications technology is broadly used and is based upon the concept of frequency re-use by the application on a series of coverage cells. Although cellular communications are now accepted into everyday life, it took many years for their development to come to mind. From developed nation to growing nation, mobile phone or cellular communications technology has been installed in all countries around the world. There is a lot of talk about the mobile phone generations. $3 \mathrm{G}$ moves on to $4 \mathrm{G}$ and then onto $5 \mathrm{G}$. Each mobile phone generation had its own aims and was able to provide different levels of functionality. There may have also been several different competing standards within the different generations. As the name indicates, cellular telecommunications technology is based around the concept of using a large number of base stations which covering a small area or cell. With each base station communicating with a reasonable number of users[25][26][27].

\section{G. Discussion}

As per requirement of CPS wireless connectivity which was mentioned in table. 4 and reviewed the exciting wireless connectivity, we summarized the Table.5. In this table, we found two wireless connectivity standards (Wireless - PON and 5G cellular network) for wireless connectivity of CPS. These two wireless standards have requirement based on latency, reliability, longevity, and data rate which is very close to CPS wireless connectivity requirement. Finally, from these two wireless standard we purposed the $5 \mathrm{G}$ wireless network for CPS wireless connectivity. $5 \mathrm{G}$ can provide the most enhanced performance than all others wireless connectivity standards based on Latency, Reliability, Longevity, and Data rate.

We review the latest published studies and papers about wireless communication technology for CPS connectivity from 2012 to 2018. The output of these reviewed papers are in the Table.5. which is determined the wireless requirements of CPS for Industry 4.0.

\section{Future Challenges AND SOlution FOR CPS WIRELESS CONNECTIVITY}

We will mention in this section some guidelines for the future of wireless communication challenges and solutions for CPS wireless connectivity in smart manufacturing. In terms of networking, signaling, interoperability, bandwidth, power consumption, and security [26], [28]-[34] [35], [36].

\section{A. Networking}

Protocols which are used in CPS wireless connectivity are anticipated to accept the protocols which are already recognized in Wireless Sensor Network connections. To increase high features for the protocols it will bring high cost, and make the protocol complicated for use. To make a new protocol not suitable task. In a particular way of thinking for the topology to be used, mesh is the highest proper selection which was used in wireless communication and to use for CPS connectivity. Dual-mesh is a protocol which is used for both wire and wireless, is very suitable for the wireless connectivity of CPS which previously used for wire applications.

\section{B. Signalling}

In the wireless communication reliability is dynamic for sending and receiving data between M to M devices and it's the role of CPS and IoT. CPS elements make connection with the server for collecting data. Or we can say server is talking with devices, or we may say elements are making connections with each other. The main point is, data should be send from point A to point B fast and reliable. We should be $100 \%$ sure that data is sending from one node to another node and will arrive to its destination all the time. When we talk about Wi-Fi standard losing of signaling happen there, but in $5 \mathrm{G}$ wireless standard there is no loss of signal and also because of their high bandwidth which make them suitable for devices communication. $5 \mathrm{G}$ radio band frequency is does not make interfering to Wi-Fi network. There is another protocol thread radio band is $2.4 \mathrm{GHz}$ which may interfering with Wi-Fi signal. Thread has no hub, it's completely distributed mesh network, with zero single point of failure, it means there is more reliability in our CPS network.

\section{Interoperability}

Presently, the main point which we want to talk about wireless connectivity is the solution of cost and how to integrate smart devices with each other. Interoperability is the focus point for markets to compete for the solution of CPS and IoT. In fact, interoperability is the ability of systems, applications, and services to work with each other to achieve the user goals and Exchange information. 5G wireless is a successful standard and has good interoperability on machine to machine devices which use wireless. ZigBee 3.0 permit a 
Table. 5 Set of values for wireless standards.

\begin{tabular}{|c|c|c|c|c|c|}
\hline \multicolumn{2}{|c|}{$\begin{array}{l}\text { Wireless Communication } \\
\text { Standards }\end{array}$} & Latency & Reliability & Longevity & Data rate \\
\hline \multicolumn{2}{|c|}{ ZigBee } & $>15 \mathrm{~ms}$ & $1 e-3$ & 100-1000 days & $20-250 \mathrm{~Kb} / \mathrm{s}$ \\
\hline \multicolumn{2}{|c|}{ Bluetooth } & $>10 \mathrm{~ms}$ & $1 e-3$ & 1-7 days & $3 \mathrm{Mb} / \mathrm{s}$ \\
\hline \multicolumn{2}{|l|}{ Wi-Fi } & $>10 \mathrm{~ms}$ & $1 e-3$ & 0.5-5 days & $54 \mathrm{Mb} / \mathrm{s}$ \\
\hline \multicolumn{2}{|c|}{ Wireless - PON } & Very low & Very High & & $155 \mathrm{Mbit} / \mathrm{s}-2.5 \mathrm{Gbps}$ \\
\hline \multicolumn{2}{|c|}{ LoRaWAN } & Based on profile & $96-99 \%$ & 10-20years & $0.25-11 \mathrm{Kbit} / \mathrm{s}$ \\
\hline \multirow{4}{*}{ 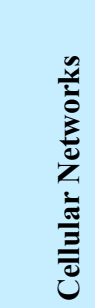 } & $2 \mathrm{G}$ & $500-1000 \mathrm{~ms}$ & High & 4-8hours in (active) and 36 days in (idle). & $100 \mathrm{~kb} / \mathrm{s}$ \\
\hline & $3 \mathrm{G}$ & $200 \mathrm{~ms}$ & High & 2-4hours in (active) and 20 days in (idle). & $384 \mathrm{~kb} / \mathrm{s}-2 \mathrm{Mb} / \mathrm{s}$ \\
\hline & $4 \mathrm{G}$ & $50-100 \mathrm{~ms}$ & Very High & 2-3hours in (active) and 12 days in (idle). & $150-450 \mathrm{Mb} / \mathrm{s}$ \\
\hline & $5 \mathrm{G}$ & $<1 \mathrm{~ms}$ & $99.99 \%$ & $>10$ years & $1 \mathrm{Gbps}-10 \mathrm{Gbps}$ \\
\hline
\end{tabular}

big range of elements to seamlessly interoperate while compare to its earlier version which fail for interoperability.

\section{Bandwidth}

Another big challenges to CPS and IoT is bandwidth consumption. Indeed, day by day lots of devices are added in smart manufacturing connectivity resulting in a massive amount of data that are produced and that need to be transported. $5 \mathrm{G}$ can be recommended in this case as it offers more bandwidth than other wireless.

\section{E. Power consumption}

In CPS technology devices are connected with each other for sharing information. Most of the devices send and receive data and they consume a huge quantity of power. An efficient connectivity is consuming low power but $5 \mathrm{G}$ and PON wireless standards are sending signal better than Bluetooth ZigBee, wife and others wireless standard. Device can easily communicate with each other for sending and receiving data.

\section{F. Security and privacy}

One important challenge for CPS wireless connectivity is cyber security. Indeed, data exchange in CPS and IoT networks raises vulnerability issues. The vital consideration for the CPS connectivity designers is hence cyber terrorists attack. Therefore, CPS and IoT needs to have policies for security. But the important entity is the cost which should be low. Various approaches provide security. One of them is crypto-primitives which should be investigated for providing authenticity (the device is not a malicious object), integrity (transmitted data is identical with the received data) and confidentiality (make data unreadable to others).

\section{CONCLUSION AND FUTURE WORK}

Cyber-Physical System is one of the key technologies for smart manufacturing. Thus, wireless connectivity in a reliable and efficient way is the key point of CPS and it is one of the importing challenges for CPS. This paper presents a detailed review of the smart manufacturing. The CPS technology as well as CPS challenges are also presented. The purpose of this research is to entertain three challenging questions and to propose a tentative solution to some-how remedies these issues. First, SMEs are build and setting-up from conventional manufacturing practices to the smart manufacturing/Industry 4.0? Second, verified and comparative analysis of the key wireless connectivity of CPS adopted for the smart manufacturing. Third, review, and suggest the wireless connectivity of CPS standards from the pool of existing standard bodies for Industry 4.0. From this review, we conclude that $5 \mathrm{G}$ wireless standard is the most adapted regarding CPS wireless requirements. This study ends by an overview of the future challenges and solution for wireless connectivity of CPS.

Future work will focus on a case study and cyber security issue of CPS wireless connectivity. The cyber security is one of the vital key challenge of wireless communication. We need to work more on cyber security issue for development of this new emerging CPS technology. 


\section{ACKNOWLEDGMENT}

This research work has been supported by SmartLink Project, Erasmus Mundus Program. The authors would like to thank SmartLink Project and Universite Lumiere Lyon2.

\section{REFERENCES}

[1] X. Li, D. Li, J. Wan, A. V. Vasilakos, C. F. Lai, and S. Wang, "A review of industrial wireless networks in the context of Industry 4.0," Wirel. Networks, vol. 23, no. 1, pp. 23-41, 2017.

[2] R. Y. Zhong, X. Xu, E. Klotz, and S. T. Newman, "Intelligent Manufacturing in the Context of Industry 4.0: A Review," Engineering, vol. 3, no. 5, pp. 616-630, 2017.

[3] A. Ahmadi, A. H. Sodhro, C. Cherifi, and Y. Ouzrout, "Evolution of 3C Cyber-Physical Systems Architecture for Industry 4 . 0 Evolution of 3C Cyber-Physical Systems Architecture for," no. May, 2018.

[4] C. Fang, X. Liu, P. M. Pardalos, and J. Pei, "Optimization for a three-stage production system in the Internet of Things: procurement, production and product recovery, and acquisition," Int. J. Adv. Manuf. Technol., vol. 83, no. 5-8, pp. 689-710, 2016.

[5] G. Schuh, C. Reuter, and A. Hauptvogel, "Increasing collaboration productivity for sustainable production systems," Procedia CIRP, vol. 29, pp. 191-196, 2015.

[6] J. Bao, Q. Wang, X. Zhen, J. Zhang, and X. Ji, "A humanmachine interaction approach of block erection schedule with three-dimensional spatial constraints," Concurr. Eng. Res. Appl., vol. 24, no. 4, pp. 359-368, 2016.

[7] J. Soldatos, S. Gusmeroli, and P. Malo, "Internet of Things Applications in Future Manufacturing," Digit. Ind., pp. 153184, 2016

[8] M. Schmitt, M. Orfgen, and D. Zühlke, "Dynamic reconfiguration of intelligent field devices by using modular software applications," IFAC-PapersOnLine, vol. 28, no. 3, pp. 561-566, 2015.

[9] S. Kong, F. Application, and P. Data, “( 12 ) United States Patent," vol. 2, no. 12, pp. 12-15, 2011.

[10] S. Bougouffa, K. Meßmer, S. Cha, and E. Trunzer, "Industry 4 . 0 Interface for Dynamic Reconfiguration of an Open Lab Size Automated Production System to Allow Remote Community Experiments," pp. 2-6, 2017.

[11] A. Ahmadi, C. Cherifi, and V. Cheutet, "A Review of CPS 5 Components Architecture for Manufacturing Based on Standards," Ski. Int. Conf. Software, Knowledge, Intell. Manag. Appl., 2017.

[12] R. Dou and G. Nan, "Optimizing sensor network coverage and regional connectivity in industrial IoT systems," IEEE Syst. J., vol. 11, no. 3, pp. 1351-1360, 2017.

[13] J. D. Contreras, J. I. Garcia, and J. D. Pastrana, "Developing of industry 4.0 applications," Int. J. Online Eng., vol. 13, no. 10, pp. 30-47, 2017.

[14] Y. Liu, Y. Peng, B. Wang, S. Yao, Z. Liu, and A. Concept, "Review on Cyber-physical Systems," vol. 4, no. 1, pp. 2740, 2017.

[15] Y. Ashibani and Q. H. Mahmoud, "Cyber physical systems security: Analysis, challenges and solutions," Comput. Secur., vol. 68, pp. 81-97, 2017.

[16] N. Jazdi, "Cyber physical systems in the context of Industry 4.0," 2014 IEEE Autom. Qual. Testing, Robot., pp. 2-4, 2014.
[17] V. Roblek, M. Meško, and A. Krapež, “A Complex View of Industry 4.0," SAGE Open, vol. 6, no. 2, 2016.

[18] B. Vogel-Heuser and D. Hess, "Guest Editorial Industry 4.0 - Prerequisites and Visions," IEEE Trans. Autom. Sci. Eng., vol. 13, no. 2, pp. 411-413, 2016.

[19] J. Lee, B. Bagheri, and H. A. Kao, "A Cyber-Physical Systems architecture for Industry 4.0-based manufacturing systems," Manuf. Lett., vol. 3, pp. 18-23, 2015.

[20] L. Thames and D. Schaefer, "Software-defined Cloud Manufacturing for Industry 4.0," Procedia CIRP, vol. 52, pp. 12-17, 2016.

[21] A. Varghese and D. Tandur, "Wireless requirements and challenges in Industry 4.0," Proc. 2014 Int. Conf. Contemp. Comput. Informatics, IC3I 2014, pp. 634-638, 2014.

[22] W. A. Khan, L. Wisniewski, D. Lang, and J. Jasperneite, "Analysis of the requirements for offering industrie 4.0 applications as a cloud service," 2017 IEEE 26th Int. Symp. Ind. Electron., pp. 1181-1188, 2017.

[23] A. Burg, A. Chattopadhyay, and K.-Y. Lam, "Wireless Communication and Security Issues for Cyber-Physical Systems and the Internet-of-Things," Proc. IEEE, vol. 106, no. 1 , pp. 38-60, 2018

[24] G. Shi and K. Li, "Signal Interference in WiFi and ZigBee Networks," pp. 9-27, 2017.

[25] http://vitorr.com, "Evolution of Mobile Communication from 1(G) to 4G, 5G, 6G, 7G ...," Http://Vitorr.Com/PostDetails.Php?Postid=2615, vol. 8, no. 3, pp. 193-197, 2017.

[26] ITU-T, "Impact of M2M communications and non-M2M mobile data applications on mobile networks," no. June, p. 90, 2012.

[27] "5G Basics 2017," 2017.

[28] J. Liu, C. Zhang, and Y. Fang, "EPIC: A Differential Privacy Framework to Defend Smart Homes Against Internet Traffic Analysis," IEEE Internet Things J., vol. 5, no. 2, pp. 1206-1217, 2018.

[29] H. Ning, H. Liu, and L. T. Yang, "Cyberentity security in the internet of things," Computer (Long. Beach. Calif)., vol. 46, no. 4, pp. 46-53, 2013.

[30] J. Bugeja, A. Jacobsson, and P. Davidsson, "On Privacy and Security Challenges in Smart Connected Homes," 2016.

[31] S. S. I. Samuel, "A review of connectivity challenges in IoTsmart home," 2016 3rd MEC Int. Conf. Big Data Smart City, ICBDSC 2016, pp. 364-367, 2016.

[32] B. L. Risteska Stojkoska and K. V. Trivodaliev, "A review of Internet of Things for smart home: Challenges and solutions," J. Clean. Prod., vol. 140, pp. 1454-1464, 2017.

[33] U. Raza, P. Kulkarni, and M. Sooriyabandara, "Low Power Wide Area Networks: An Overview," IEEE Commun. Surv. Tutorials, vol. 19, no. 2, pp. 855-873, 2017.

[34] A. A. Khan, M. H. Rehmani, and M. Reisslein, "Cognitive radio for smart grids: Survey of architectures, spectrum sensing mechanisms, and networking protocols," IEEE Commun. Surv. Tutorials, vol. 18, no. 1, pp. 860-898, 2016.

[35] M. A. Shah, Y. Li, and A. H. Sodhro, "Energy-efficient adaptive transmission power control for wireless body area networks," IET Commun., vol. 10, no. 1, pp. 81-90, 2016.

[36] A. H. Sodhro, S. Pirbhulal, A. K. Sangaiah, S. Lohano, G. H. Sodhro, and Z. Luo, "5G-based transmission power control mechanism in Fog computing for internet of things devices," Sustain., vol. 10, no. 4, pp. 1-17, 2018. 